\title{
Versatile computerized system for tracking and analysis of water maze tests
}

\author{
TATYANA V. MUKHINA, SERGEI O. BACHURIN, \\ NADEJDA N. LERMONTOVA, and NIKOLAI S. ZEFIROV \\ Institute of Physiologically Active Compounds, Russian Academy of Sciences, Moscow, Russia
}

\begin{abstract}
A crucial step in the estimation of properties of compounds in behavioral experiments is the quantification and description of the different effects observed. The goal of the present work was the automation of the Morris water maze test, one of the most popular behavioral methods for the study of animal memory. An original system was developed that provides fast and accurate tracking of animals, storage of the results in the database and video archive and a means of analyzing the results. This computerized version of the Morris water maze test permits the quantification of such vague characteristics of cognitive function as the "directionality" of search of the hidden platform after a standard training series. The suggested parameters made it possible to discriminate cognitive properties of the novel compounds from other behavioral effects affecting escape latency. The effectiveness of this system was demonstrated in two experiments with neurochemically lesioned and drug-treated rats.
\end{abstract}

Behavioral experiments using animals are very important for estimating the cognitive effects of chemical compounds. Accurate quantification, description, and analysis of the results of such experiments is crucial. One of the frequently used methods for estimation of memory and cognition functions of animals is the Morris water maze test (Morris, 1981; Steele \& Morris, 1999). In this test, rats' capability to find a platform hidden beneath the water surface in a round swimming pool is estimated. As a rule, the time it takes a rat to find the hidden platform (escape latency) after previous training is determined as a main measure of memory. Apparently, escape latency is the only parameter that can be registered manually. This is completely insufficient for characterization of complex behavior of the animal, so computerization of the test is almost unavoidable. Such a computerized system must provide fast and accurate tracking with realtime computation of certain parameters (so that the researcher can make some decisions during the experiment), optimal organization of a large data array, automatic creation of a video archive, and a means for analyzing the results obtained using different criteria. In our work on newly synthesized compounds as potential neuroprotectors or cognition enhancers for prevention and treatment of neurodegenerative disorders such as Alzheimer's disease, we could not find a system meeting all these require-

This research was supported by a grant from the International Science and Technology (Project 1512) and partially supported by the Russian Foundation for Basic Research (Project 00-04-48398) and the Young Scientists Program of the Russian Academy of Sciences. Correspondence should be addressed to T. V. Mukhina, Laboratory of Neurochemistry, Institute of Physiologically Active Compounds, Russian Academy of Sciences, 142432, Chernogolovka, Moscow, Russia (e-mail: mukhina@ipac.ac.ru) ments, so we initiated development of an automated system, "Behavioral Vision," for registering the motion of experimental animals in the Morris water pool test. Thus, the goal of the present work was automation of the Morris water pool test. A description of the newly developed computer system is provided, as well as two experiments demonstrating its efficacy.

\section{TRACKING SYSTEM}

\section{Hardware}

The hardware should provide a resolution high enough for a viewer to be able to detect a small (at least $5 \mathrm{~cm}$ ) white object (rat) on a large black surface (a round black pool $1.8 \mathrm{~m}$ in diameter, $0.5 \mathrm{~m}$ in height, and filled with water to the depth of $0.3 \mathrm{~m}$ ). The tracking speed should be sufficient for real-time signal processing. The hardware should also permit the creation of a video archive and the adjustment of the video path parameters for optimization of the tracking conditions.

The scheme of the video system is presented in Figure 1. An analog black-and-white (B/W) video camera was selected as a source of video signal, since its resolution $(600$ lines or $\sim 3 \mathrm{~mm}$ ) was adequate. To enable adjustment of the size of the image, a camera with a zoom lens was chosen. The camera was connected to an S-VHS video cassette recorder (VCR), which was preferred because of its higher resolution in comparison with standard VHS. To perform automatic recording and playback, the VCR should have an RS-232 interface. A B/W monitor made it possible to conveniently observe the motion of the animal on a larger screen. The signal from the camera was sent into a framegrabber installed in the hard drive of the computer. The framegrabber should not load the processor and catch the frames in real time, namely, up to 25 frames per 


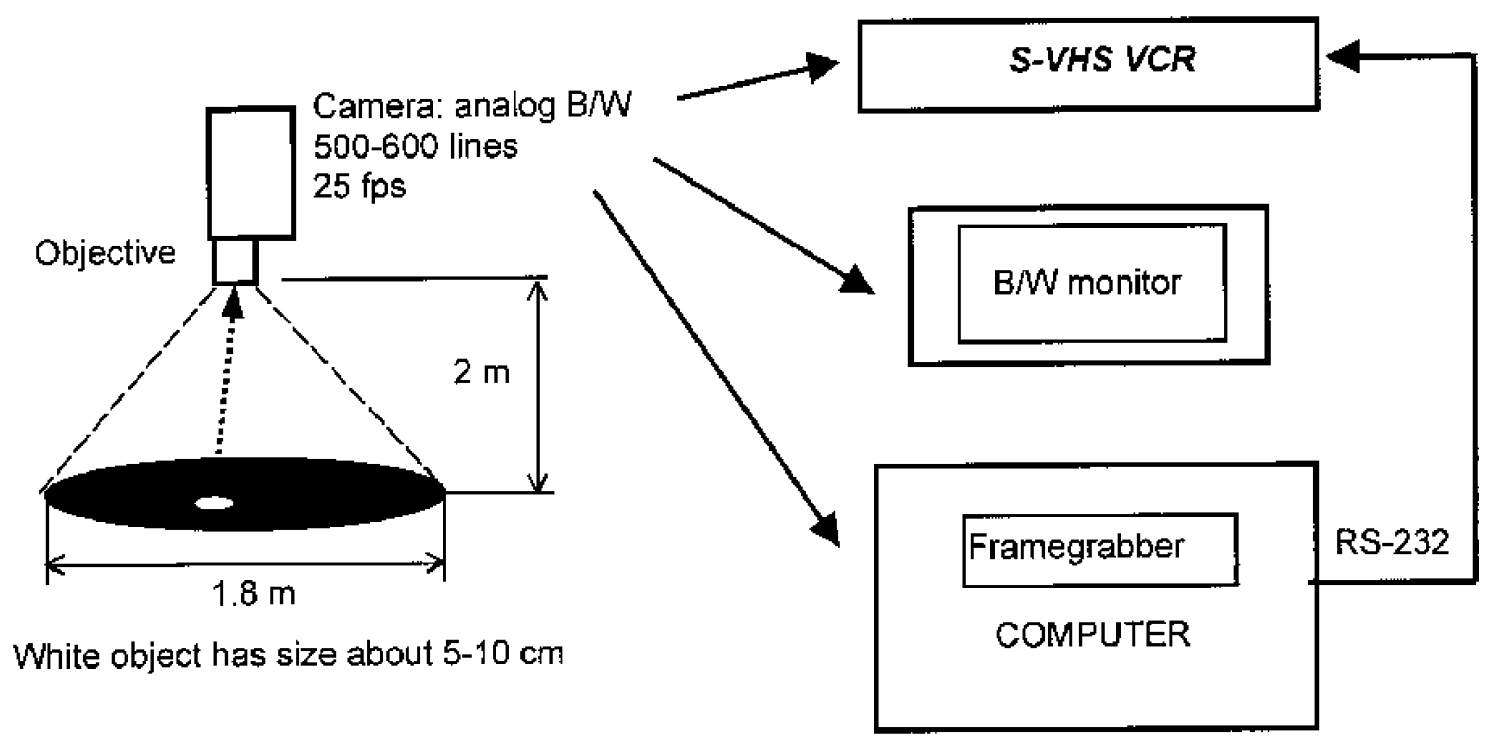

Figure 1. Scheme of the video system.

second. The image to be analyzed was sent into the RAM of the computer, where its processing enabled us to have a "live" video window on the computer display.

\section{Software}

The software should provide fast and accurate tracking (detection of an object on a flashing water surface), storage of the results in a designated database and video archive, and a means for analyzing the data obtained in order to capture both standard and unusual characteristics of the animals' behavior. Automatic setting of the position and size of all zones of interest where the object is tracked would be very useful as well.

"Behavioral Vision" operates under Windows 9x/NT/ 2000 and offers a user-friendly interface. The program has a well-developed menu structure that allows acquisition, visualization, and analysis of the data, definition of various configuration parameters, and so on. The system is maximally automated and easy to use, making it possible for the experimenter to concentrate on the behavioral characteristics of interest rather than on experimental routines. The program also has a comprehensive help system and detailed documentation.

Data storage and management. The program features a flexible database management system instead of storage in files of different types. The structural elements of this database are "tracks" and "experiments." "Tracks" represent single animal trajectories containing temporal and spatial information about their motion; "experiments" store sets of tracks obtained in the same or different conditions. All the information about the experiment, including position of the track on the videotape, is stored in the database, so the tracks can be played back or digitized anew (if required). The database also contains other information, such as the compound used for treatment of each animal, dose, animal sex, and any researcher-written text comments, so the program can be used as a laboratory journal. Tracks can be edited and moved between existing experiments in the database, and both tracks and experiments can be deleted from the database.

Zones' definition. The program automatically detects the borders of the pool and other analyzed zones (e.g., platform), taking into account perspective and geometric distortions (Figure 2A). The automatic detection is based on optical contrast: for example, a white border $\sim 40$ $50 \mathrm{~mm}$ wide around a black pool helps to define its boundary; a white circle set on the black platform to indicate its position (the platform size is set in the configuration), and so on. Such a principle of zone detection seems to be easier and much more reliable than definitions using different drawing tools (rectangle, circle, etc.), as organized in some other programs. Calibration of the system (conversion of distances in the digitized video image to real distances, e.g., in millimeters) is performed automatically at the definition of the pool boundary (or loading it from a special desktop file if geometrical parameters of the pool were stored in it previously) using values of real pool diameter, width of its border, position of the camera, and water level (specified by a user in the program settings). The defined zone parameters, tracking parameters (target zone, stopping conditions, etc.), and other program settings, can be saved into special files and then easily read from them. Thus it is unnecessary to make zone definitions every time you start the program; you can load geometrical parameters from the corresponding desktop file. It is very important to set the removed platform to the same position where it was, for example, during the training. Live video provides two options. First, you can load the content of the selected desktop file, display it in the live video window (as shown in Figure 2A), place a white circle on the black platform (to make it visible on the black background), and move it manually to the po- 

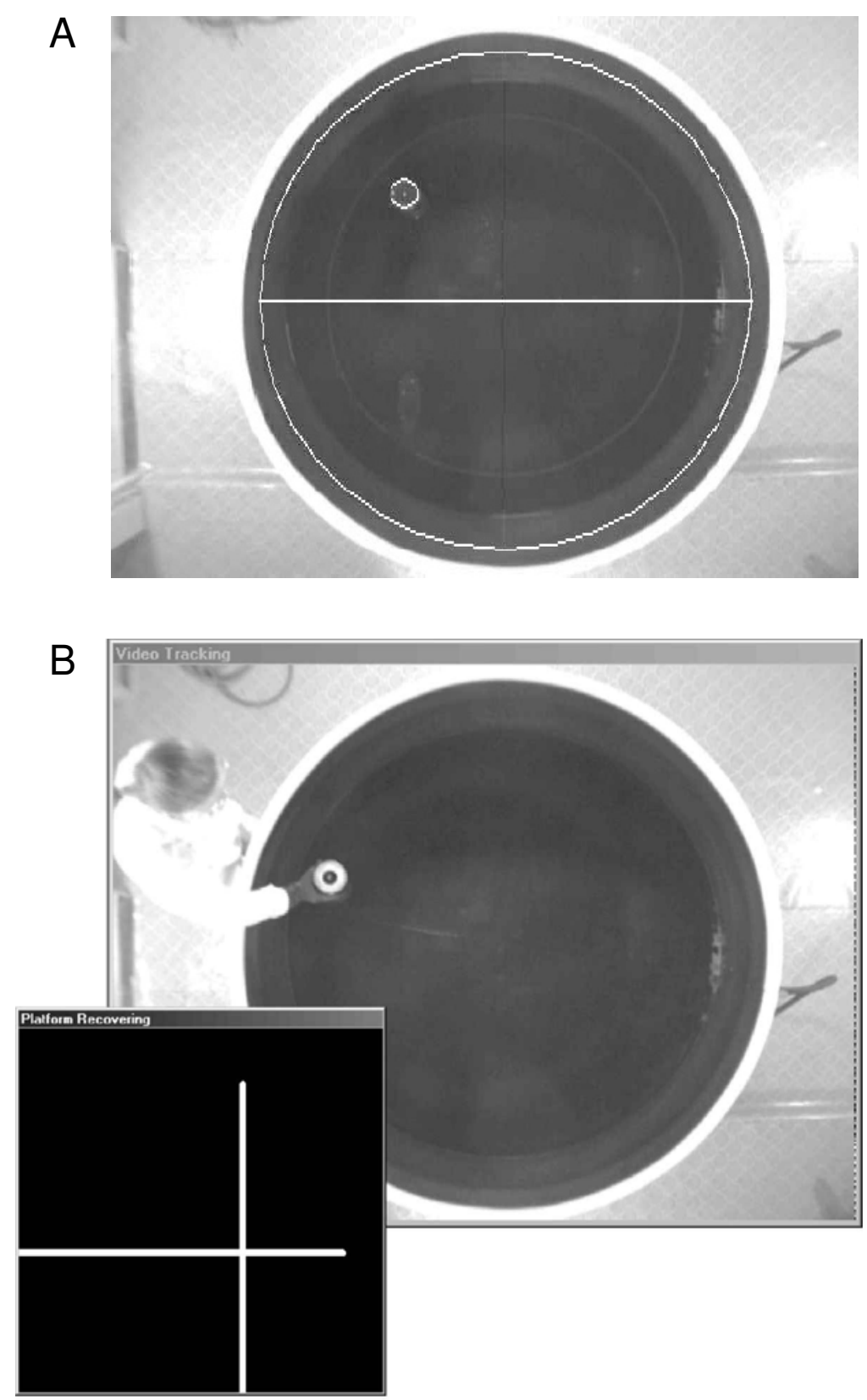

Figure 2. Definition of zone borders. (A) Result of zone detection; (B) restoration of the platform position. Free ends of the white crest in Figure 2B indicate the directions in which the platform should be moved; the distances between these ends and window borders are proportional to the corresponding horizontal and vertical distances between the current and target platform positions.

sition shown on the computer monitor without updating the video image. Second, automatic recovering of the platform position is available (Figure 2B). This procedure asks for the desktop file and then computes direction and distance to move the platform so that its position relative the pool is the same as specified in the file. The program visually indicates to the user where he/she should place the platform.
Object tracking. Since our goal is the automation of Morris water maze test, "Behavioral Vision" tracks only one object in the pool. In the tracking process, all relevant information is presented in real time in separate windows ("live" video, trajectory, elapsed time, speed, direction vector, etc.) so that the experimenter can make some conclusions immediately. The object can be tracked either by searching the complete arena or smaller region 
(scan window). A "streaming" mode where tracking is started automatically at the appearance of an animal in the pool and stopped at specified conditions is also available in our system, which is very convenient for testing large numbers of animals.

The detection and tracking of the object on a water surface is often complicated by numerous flashes from the water, animal excrement, and sawdust falling into the pool during the experiment. These additional objects hinder the search. This problem was solved in two ways (Figure 3): (1) by incorporating programmable correction of the video path in the framegrabber, which significantly decreased computations performed during object detection but did not remove all parasite white spots, and (2) by using elements of artificial intelligence in the detection algorithm that take into account the object prehistory to estimate its current and future position. All found white spots are formed by pixels with the userdefined brightness threshold. The construction of the unit and the special algorithm of processing of the image make it possible to solve this problem successfully, so the tracking process is insensitive to illumination and never crashes (Figure 4). When the animal is found, its position can be adjusted using an erosion algorithm to discard the tail and other protruding parts of the animal to obtain a more accurate estimation of the rat's position.

Analysis. During the tracking analysis a large number of parameters can be calculated: escape latency, speed, total time and trajectory length or their parts within different zones, criteria characterizing development of the strategy (distance to platform, Whishaw "alley" index), and new indices characterizing directionality of tracks (straightness index, angle characteristics) and decreases in mobility (index of passive swimming). The rats can also be subdivided into three groups according to their escape latency by the user-defined time intervals. The user can create various zones and combine them to compute the parameters for the parts of the trajectories. This is very useful for the analysis of probe trials (when, after the series of training trials, the animals are allowed to swim for a fixed time without the platform). The results of the analysis can be presented graphically in different forms. Single tracks or whole experiments can be analyzed in our program. The analysis can be applied to arbitrary combinations of tracks or/and experiments. It can be useful when, for example, control groups from different experiments need to be analyzed together. The selected $\operatorname{track}(\mathrm{s})$ or whole experiments can be grouped for the analysis by compound, dosage, and directionality. Primary statistical analysis of the data obtained is performed within the program. For more complex statistics, the data can be exported into other programs, such as Microsoft Excel, Statistica, and so on.

\section{PARAMETERS}

Here we will describe only the novel and more elaborated indices. Daily training of rats using two trials a day with the hidden platform in the same position (i.e., the standard water maze acquisition task, Grigoryan, Hodges,

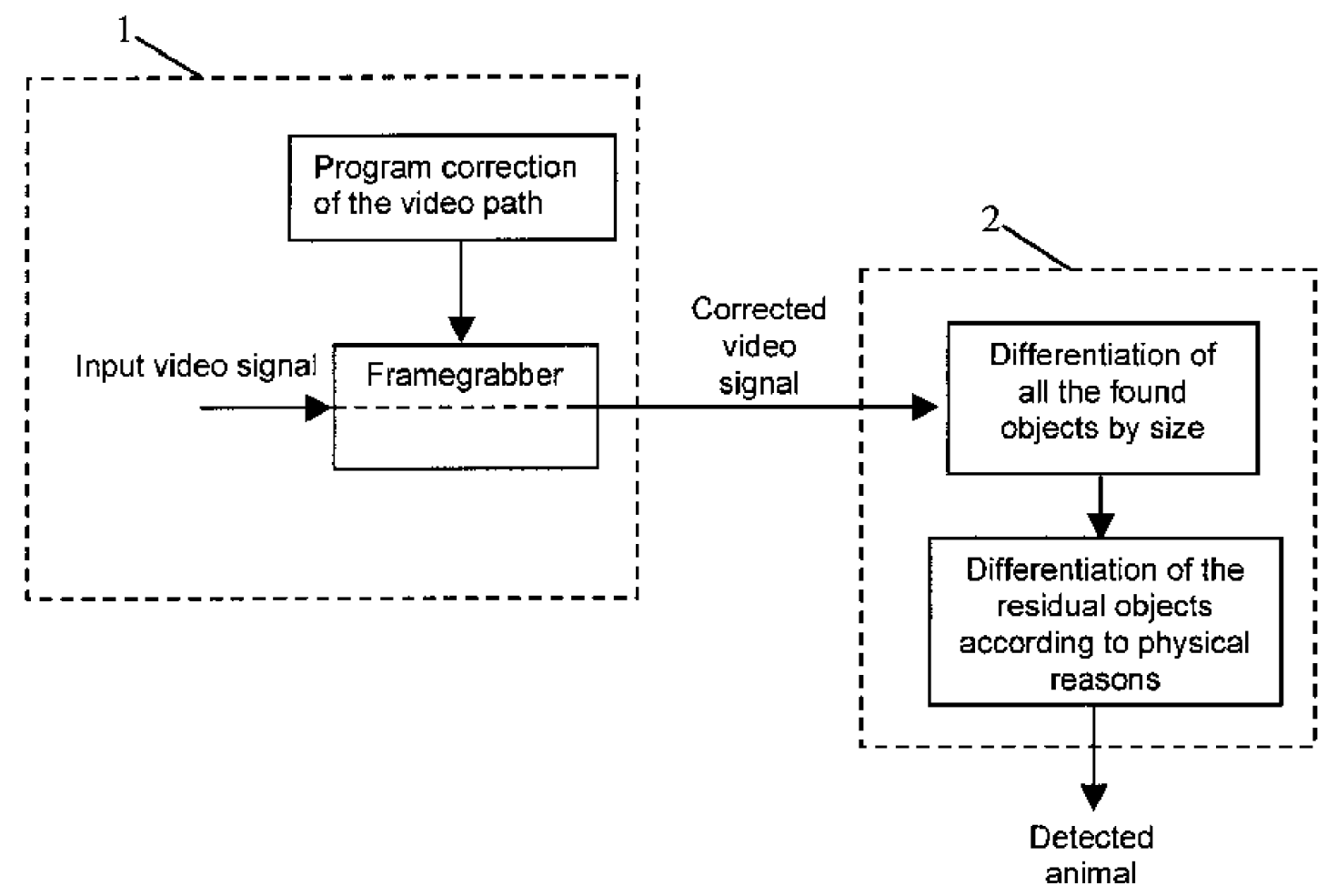

Figure 3. Scheme of the object detection algorithm. 


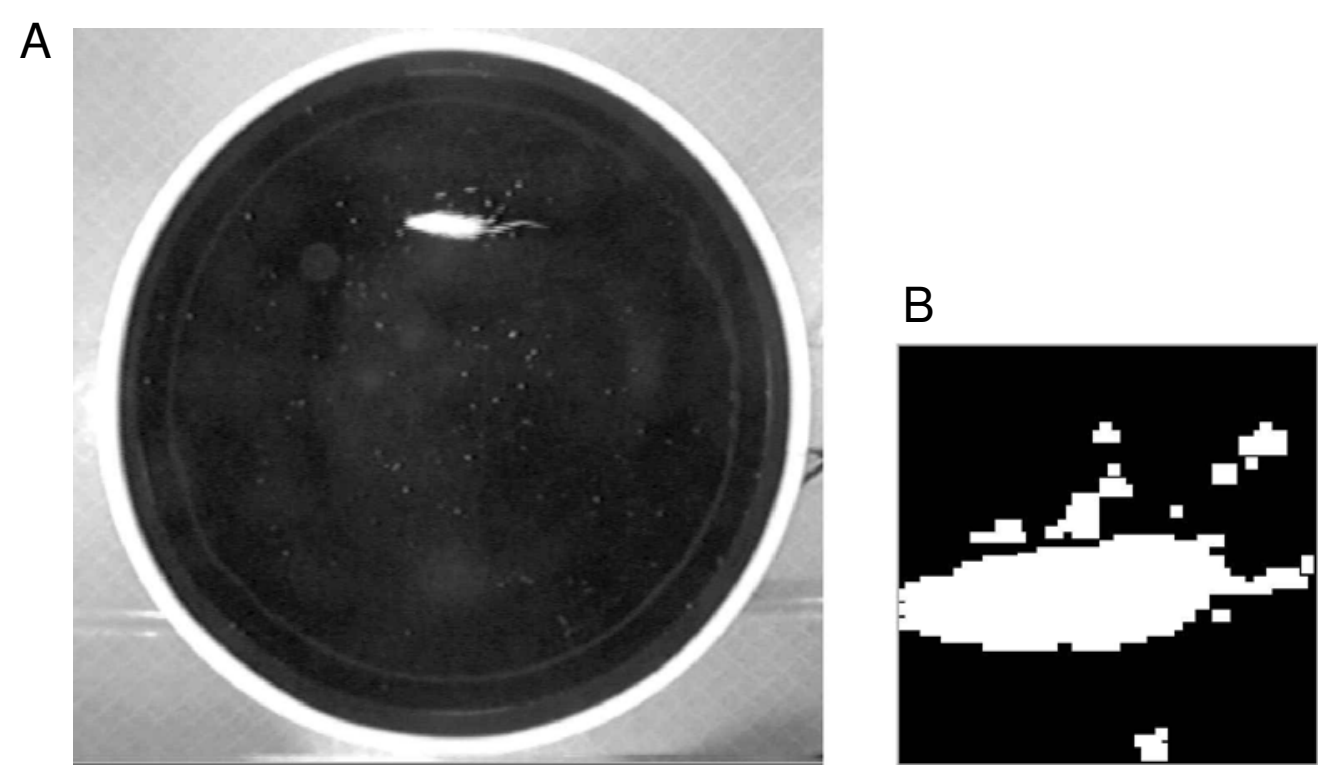

Figure 4. Current video frame (A) and corresponding result of the object detection. (B) Large white object with tail is a rat; small white spots are flashes on the water.

Mitchell, Sinden, \& Gray, 1996) leads to the development in rats of search behavior strategies. Swimming near the pool walls, typically observed for all groups of rats at the beginning of the test, changes for some of them during the training to a more or less directed search for the hidden platform (e.g., the control and AF64A+ medicine-treated groups in Figure 5); in other groups, most of the rats may fail to find the platform even at the late training stage (e.g., the AF64A-injected rats in Figure 5). Often it is impossible to differentiate the escape performance of the rats treated with the compounds studied using only the time required to find the hidden platform, since the difference between their behavior evaluated by this parameter is not statistically significant.

The animals' ability to generate the behavior required for performing the task is connected with development of an "optimal" strategy of the search and, hence, more "directed" shape of the trajectories. In place of the vaguely formulated term directionality, we proposed the terms straightness index and vector characteristics. The straightness index is calculated as a ratio between the trajectory length and the distance from the track start to the platform center (i.e., deviation of the trajectory from the global reference vector connecting start position and platform). Direction of the vector of speed of the animal seems to be one of the most important characteristics of directionality, so we used several forms of weighted angles and rates of their variation. The suggested angular characteristics include a path-averaged sum

$$
\beta=\sqrt{\frac{\sum_{i=1}^{N}\left(\beta_{i}^{2} \cdot \Delta l_{i}\right)}{L}}
$$

of the angles $\beta_{i}$ between the vectors of speed and direction from the current point to the platform in each point of the trajectory having total length $L$ ( $N$ is the number of points in the trajectory), sum of angles $\alpha_{i}$ between direction of speed in each point of the trajectory and global reference vector

$$
\alpha=\sqrt{\sum_{i=1}^{N}\left(\left|\alpha_{i}\right| \cdot \Delta l_{i}\right)},
$$

and dynamic of the change in the turn angle relative to the reference vector

$$
\Delta \alpha=\sqrt{\sum_{i=1}^{N}\left(\left|\alpha_{i}-\alpha_{i-1}\right| \cdot \Delta l_{i}\right)},
$$

where $\alpha_{i}$ and $\alpha_{i-1}$ are the angles in two adjacent points of the trajectory, and $\Delta l_{i}$ is the increment of its length in the $i$ th point. The angles in each point are multiplied by the corresponding length increment $\Delta l_{i}$ to exclude from calculation spontaneous changes of the angle at the points where a rat is not moved.

In addition, we analyzed a criterion characterizing the strategy of the search for the hidden platform, the ситиlative distance to platform,

$$
\sqrt{\sum_{i=0}^{N}\left|R_{i}\right|}
$$

(where $N$ is the number of points in the trajectory and $R_{i}$ is a distance between the trajectory and platform position) used earlier for the recognition of subtle differences between swim strategies (Dalm, Grootendorst, de Kloet, \& Oitzl, 2000); the square root here is used to decrease the dynamical range of this parameter. Another index, similar to that of Cain, Ighanian, and Boon, (2000) and Whishaw, Cassel, and Jarrard (1995), the percentage of 


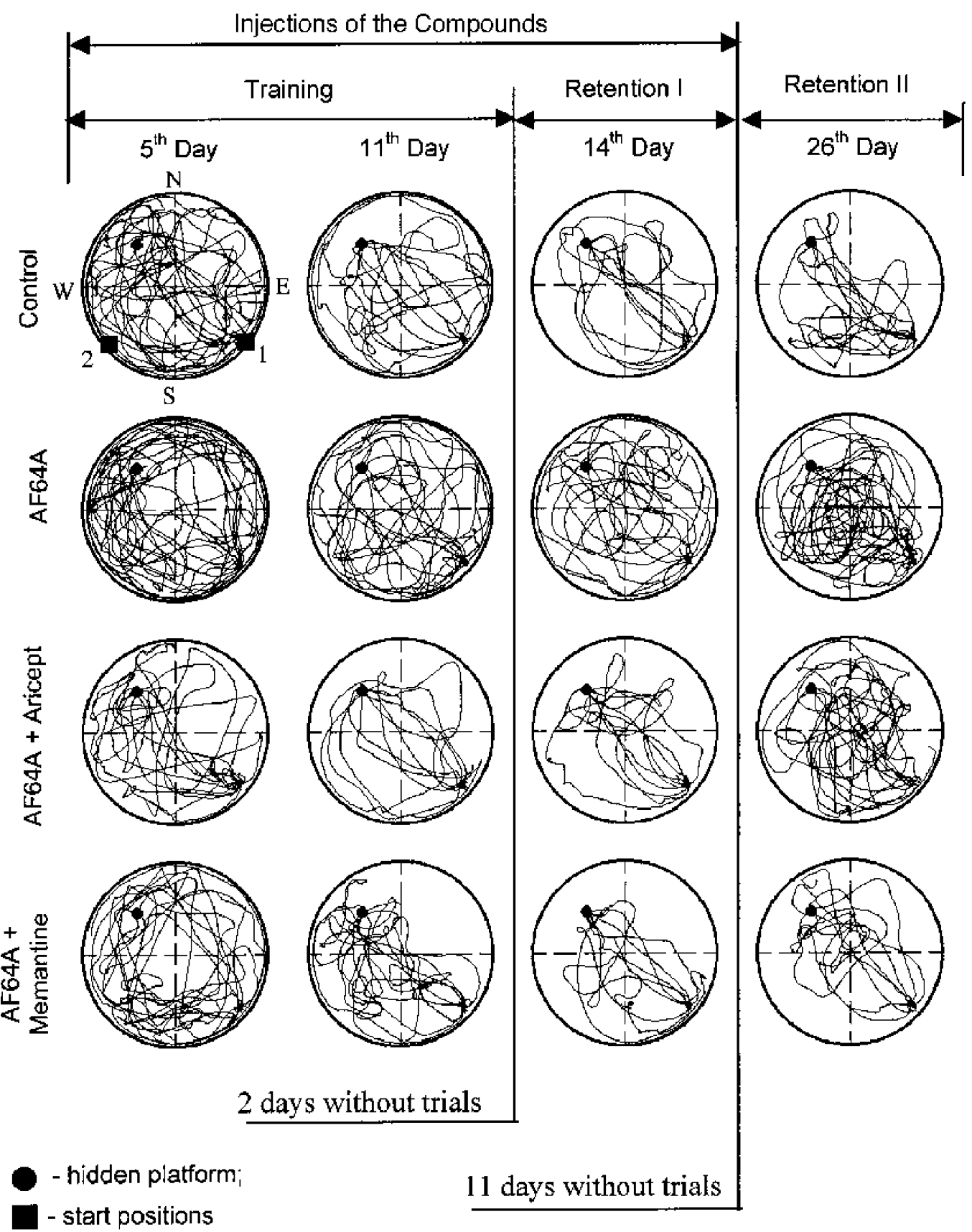

Figure 5. Effect of Aricept and Memantine on the learning and memory of experimental rats in comparison with control and AF64A-treated groups of rats. Each swim pattern presents superimposed tracks of all rats in the corresponding group. On both the training and retention days they received two trials/day with the hidden platform in the same position. No trials were performed on Days 12 and 13 and within a period from Day 15 to Day 26 (the days are enumerated starting from Day 1 of the training). No probe trials were conducted in this case.

the trajectory within a corridor centered on a reference vector, was used to assess directionality characteristics.

Some compounds can decrease rats' movement. For example, during the search for the target, a rat can stop or move very slowly in some parts of the trajectory. Such a behavior may be connected with sedative effect, de- crease of motivation, and so on, leading to an increase in the escape latency. A parameter such as average speed is insufficient for estimating this effect. We suggested an index of passive swimming, calculated as a ratio of the time spent with the speed lower than a threshold value to the time of swimming with the larger speed. The thresh- 
old value is set by the user (e.g., $1-5 \mathrm{~cm} / \mathrm{sec}$ ). In addition, "active" time (i.e., time of swimming with speed exceeding the threshold) can be computed.

\section{EXPERIMENTAL SETUP}

Two experiments were performed to demonstrate the efficacy of the suggested criteria for estimating the effect of selected compounds on the learning and retention ability of rats. In the first experiment (Figures 5 and 6), the developed program and parameters were used to estimate the effects of the well-known anti-Alzheimer's medicines Aricept and Memantine on the long-term memory of rats with a form of Alzheimer's caused by neurotoxin AF64A (Bachurin et al., 1999; Fisher \& Hanin, 1986; Lermontova, Lukoyanov, Serkova, Lukoyanova, \& Bachurin, 1998; Walsh \& Opello, 1994). In the second experiment, the compound did not affect the learning and retention abilities of the intact rats (i.e., those without neurotoxin AF64A), but did decrease their swimming activity (Figures 7 and 8 ). In both experiments, the rats injected with the compounds received the standard acquisition procedure: two trials/day from two different starting positions with a 10 -min intertrial interval and
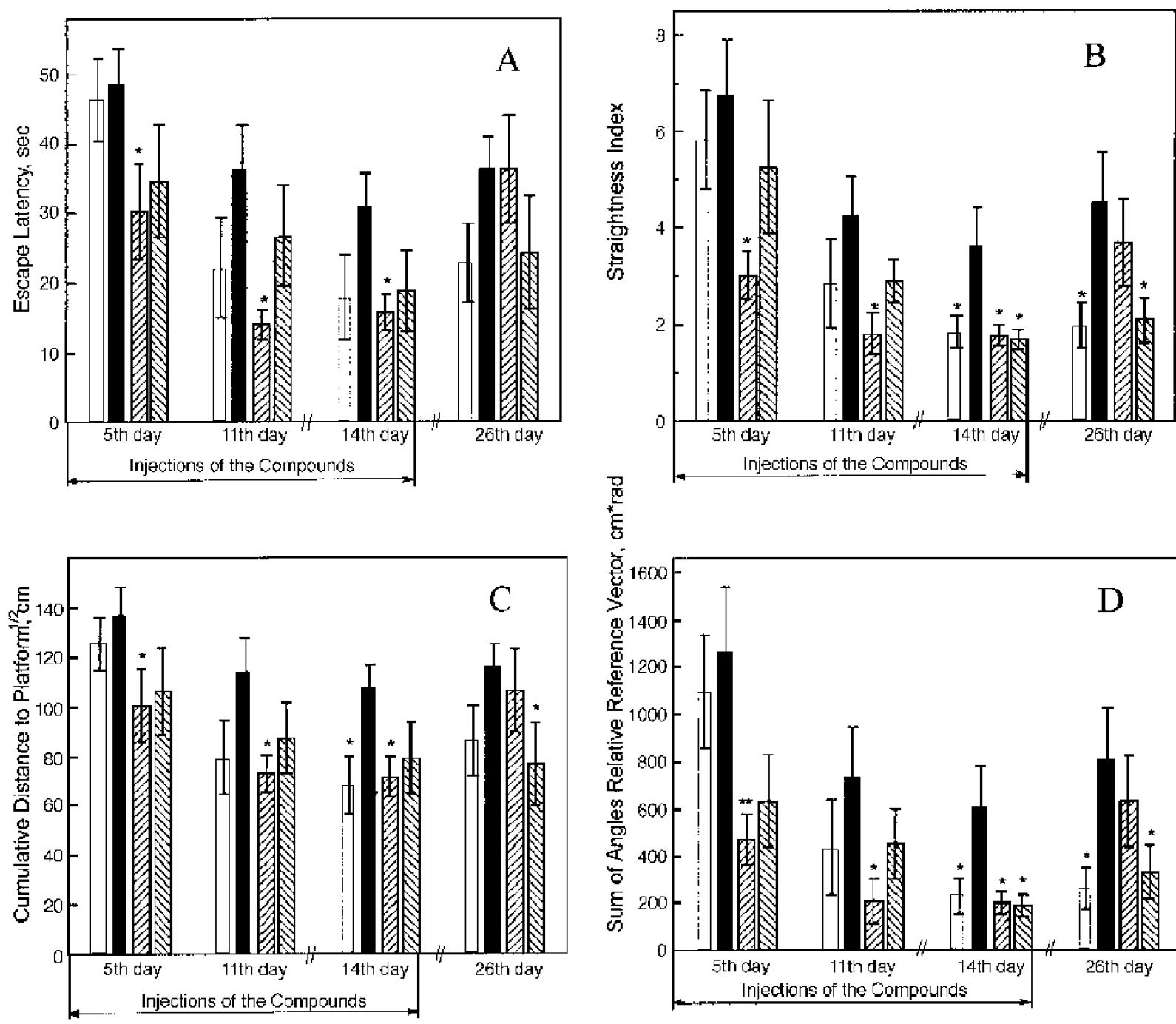

$$
\begin{aligned}
& \square \text { Control, } n=10 \\
& \mathscr{W} \text { AF64A + Aricept, } n=10
\end{aligned}
$$

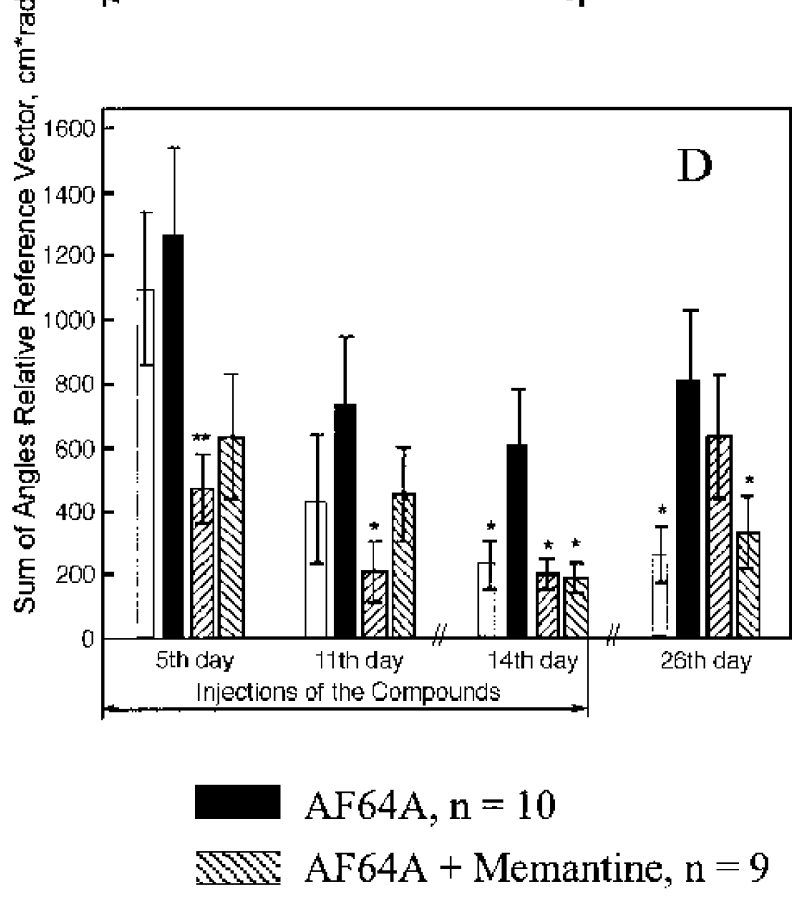

\section{/ No trials}

Figure 6. Comparison of estimation of the compounds effect using escape latency (A), straightness index (B), cumulative distance to platform (C), and sum $\alpha$ of angles between vector of speed and global reference vector in each point of the trajectory (D) in the training and retention trials. $n$, number of rats in each group. $* p<.05 . \quad * * p<.01$ vs. AF64A group. 


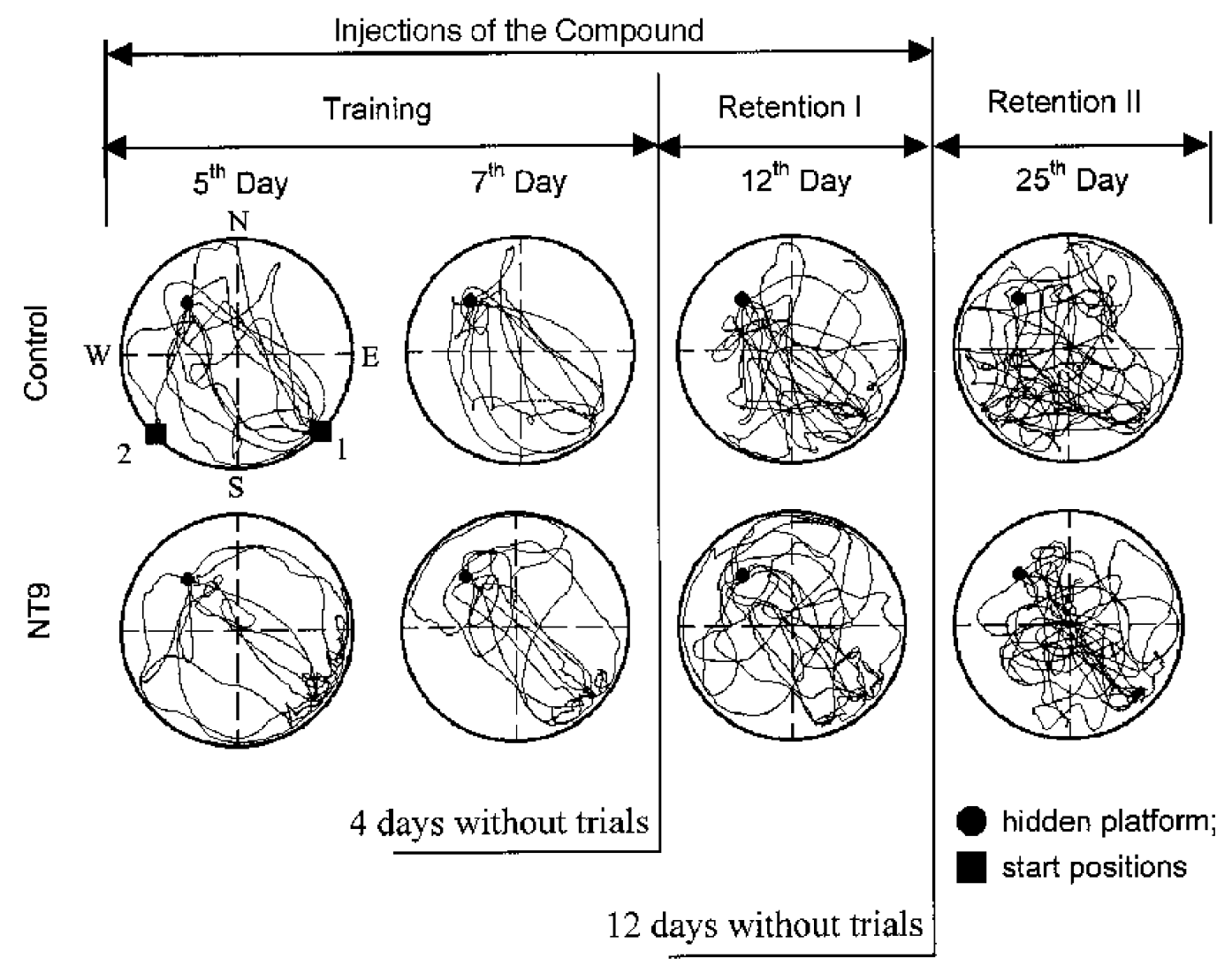

Figure 7. Effect of NT9 on the learning and memory of experimental rats in comparison with control rats. Each swim pattern presents superimposed tracks of all rats in the corresponding group. On both the training and retention days they received two trials/day with the hidden platform in the same position. No trials were performed from Day 8 to Day 12 and from Day 14 to Day 25 (the days are enumerated starting from Day 1 of the training). No probe trials were conducted in this case.

the hidden platform in the same position (in the center of the NW quadrant, $1.5-2.0 \mathrm{~cm}$ below the water surface). Rats were placed in the pool, facing the wall, and allowed to swim for $1 \mathrm{~min}$. If they found the platform within this time, they were allowed to remain there for $10 \mathrm{sec}$; if they failed to find the platform, they were guided to and placed on it for $10 \mathrm{sec}$. The procedure was the same on all the training and retention days. After finishing the series of the training trials, all the rats had a rest for several days ( 2 and 4 days for Experiments 1 and 2, respectively) before the "Retention I" stage at the continued injections. Then the compounds injections were stopped, and the animals had a longer period without the trials between Retention 1 and Retention 2 (11 and 12 days for Experiments 1 and 2, respectively). Since the first trial is the most important for the study of long-term memory, only tracks started from Position 1 were analyzed. No probe trials were performed in these experiments. An analysis of variance (ANOVA) followed by post hoc LSD comparisons was performed in the program package STATISTICA (Version 5.0). The significance level was preset at $p<.05$. Data are presented as means plus or minus standard errors.

\section{RESULTS}

The tracking patterns shown in Figure 5 clearly demonstrate task performance improvement for all rats except for the AF64A-injected group. The escape latency calculations indicate that this effect was barely visible (Figure 6A). The straightness index (Figure 6B) permits a clearer picture of the learning ability of the various groups of rats. The relationships between the groups' mean trajectory lengths are shown in Figure 6B. There was a smaller cumulative distance to the platform for the control and AF64A+Memantine groups relative to the neurotoxin-AF64A-only group (Figure 6C). Among the suggested vector characteristics, only $\alpha$ (Figure 6D) and the dynamics of change of the turn angle $\Delta \alpha$ (which has the same tendency and is not shown in Figure 6) yielded a statistically significant difference between analyzed groups. No statistically significant differences were observed between the groups of rats in the values of mean speed and index of passive swimming in this experiment.

In the second experiment (Figure 7), NT9-injected rats demonstrated a tendency to have both higher escape latency (Figure 8A) and greater cumulative distance to 

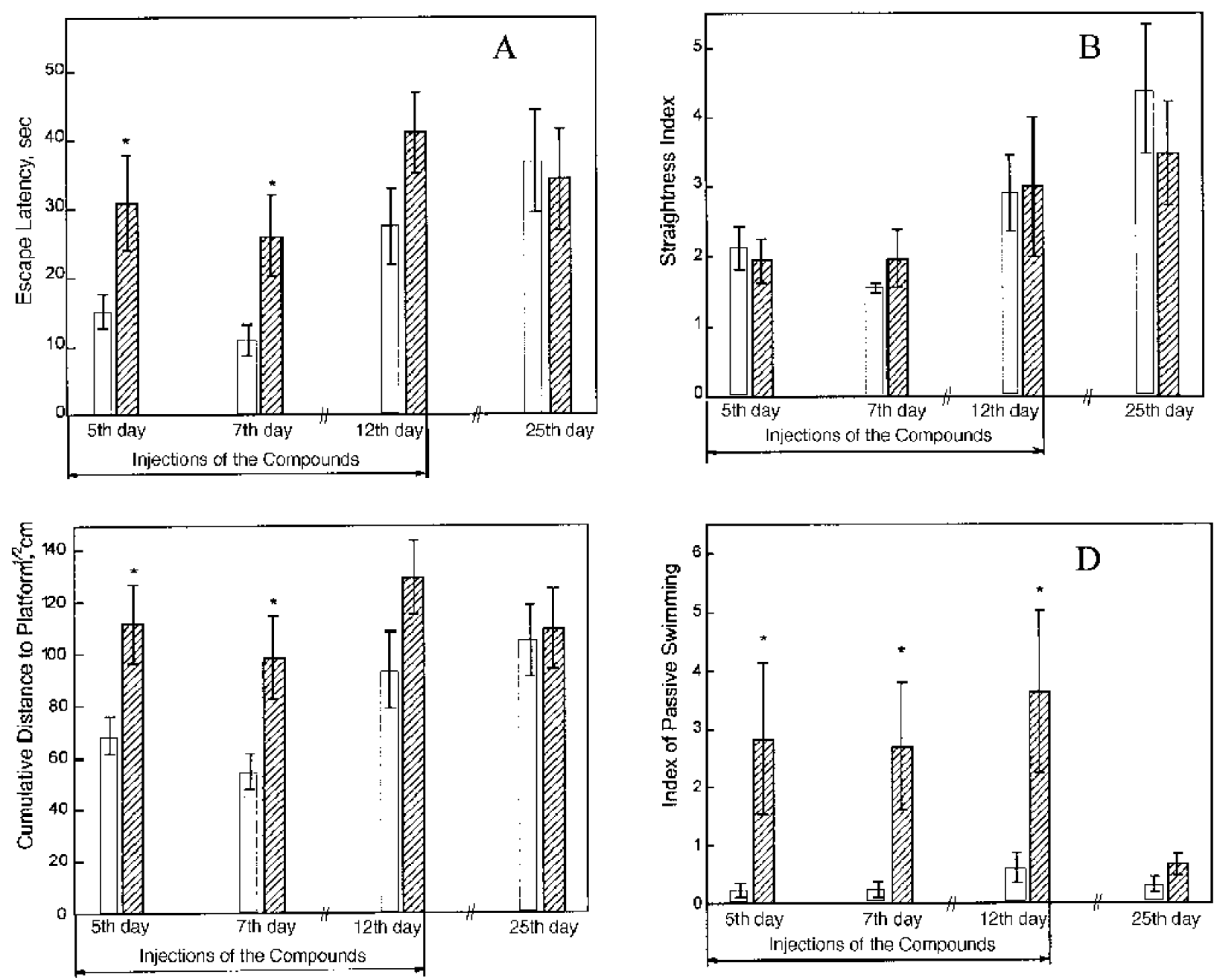

$$
\text { Control, } \mathrm{n}=10 \mathbb{W} \text { UNT9, } \mathrm{n}=10
$$

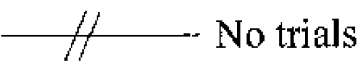

Figure 8. Comparison of estimation of the compounds effect using escape latency (A), straightness index (B), cumulative distance to platform $(C)$, and index of passive swimming $(D)$ at the learning and retention trials. $* p<.05$ vs. control group. $n$, number of rats in each group.

the platform than the control group (Figure 8C), except for Day 25. According to these criteria, NT9-injected animals could be falsely classified as incapable of spatial learning in comparison with the control group. However, the directionality of their tracks as calculated by the straightness index (Figure 8B) and vector characteristics $\alpha$ and $\Delta \alpha$ (not shown in the figure) was always similar to that of the control rats, suggesting that NT9 had no effect on the animals' learning and retention. Since the average speed of NT9-injected rats was smaller than that of the controls (although the difference was not statistically significant), this parameter did not help us to explain the contradiction between the results obtained. The index of passive swimming revealed the effect of a decrease in activity of NT9-injected rats, which almost disappeared after the injections were discontinued (Figure 8D). Thus, this pa- rameter made it possible to quantitatively explain the increase in the escape latency and cumulative distance to the platform with directionality similar to that of the control group.

\section{DISCUSSION}

The parameters used depend on the goal of the experiment. Some of them, such as escape latency, trajectory length, mean speed of the animal, and so on, can be used for behavioral estimation of any water maze experiments (Dalm et al., 2000; Eijkenboom, Gerlach, Jork, Lowe, \& van der Staay, 2000). For example, measurements during initial trials of the water maze task may be helpful for studying rats' investigative activity. All of these parameters can be used as well for the evaluation of rat cogni- 
tive functions during training. Such parameters as search index (ratio of the times in target and start zones), time in the platform zone, time spent in different quadrants in comparison with training quadrant, and so on, are used most often for estimating trained rats' ability to remember the position of the platform, which has been removed after the training trials (Dalm et al., 2000; Eijkenboom et al., 2000).

The present results on the newly suggested parameters-straightness index, sum of the angles between direction of speed in each point of the trajectory, and global reference vector, as well as dynamics of change of the turn angle relative to the reference vector-indicate that these parameters provide a better means of capturing key elements of rat behavior in the water maze task, allowing finer discrimination of rat behaviors relative to the standard, previously used parameters of escape latency, trajectory length, speed, and cumulative distance to platform.

In particular, the straightness index and the two vector characteristics seem to be the most powerful parameters for differentiating swimming patterns on the basis of search directionality. They most adequately correlate with visual perception of the corresponding trajectories. The description of the trajectories by their lengths gives the same results as the straightness index. However, the latter seems to be more illustrative than the trajectory length itself and can be used for comparison of tracks received from different start positions in pools of different size. The path-averaged sum of the angles between the vectors of speed and direction from the current point of the trajectory to the platform did not yield adequate estimation of the visual differences between swimming patterns (as in Figure 5) for the task with the hidden platform. This parameter may be more profitably used in the analysis of the animals' ability to find the visible target.

The squared root of the cumulative distance to platform also provided good descriptions of the track patterns in certain cases. Furthermore, the index of passive swimming describes the effect of changing movement much better than the standard calculations of mean speed. This parameter is very useful especially when a compound decreases moving activity along with slight change or improvement of memory functions.

So, different parameters describe different aspects of animals' behavior. Introduction of our new parameters makes it possible to estimate more accurately the effects of compounds on animal behavior in the Morris water maze.

In sum, a new robust computerized system was developed for tracking rat behavior in the Morris water pool. This system allows automatic digitizing of the animals' trajectories, data storage in a specially developed database, maintenance of a video archive, and the means for a comprehensive analysis of the results obtained.

The computerized system also permits the use of a new set of parameters-straightness index, two vector parameters, and an index of passive swimming. Combining these new parameters with the standard ones (escape latency, speed, cumulative distance to platform) was shown to provide more finely tuned estimation of rat behavior in the water maze task.

\section{REFERENCES}

Bachurin, S., Oxenkrug, G., Lermontova, N., Afanasiev, A., Beznosko, B., Vankin, G., Shevtzova, E., Mukhina, T., Serkova, T. (1999). $N$-Acetylserotonin, melatonin and their derivatives improve cognition and protect against $\beta$-amyloid-induced neurotixicity. In B. Trembly \& W. Slikker, Jr. (Eds.), Neuroprotective agents: Fourth international conferences (Annals of the New York Academy of Sciences, Vol. 890, pp. 155-166). New York: New York Academy of Sciences.

Cain, D. P., Ighanian, K., \& Boon, F. (2000). Individual and combined manipulation of muscarinic, NMDA, and benzodiazepine receptor activity in the water maze task: Implications for a rat model of Alzheimer dementia. Behavioural Brain Research, 111, 125-137.

Dalm, S., Grootendorst, J., de Kloet, E. R., \& Oitzl, M. S. (2000). Quantification of swim patterns in the Morris water maze. Behavior Research Methods, Instruments, \& Computers, 32, 134-139.

Eijkenboom, M., Gerlach, I., Jork, R., Lowe, D., \& Van der StaAy, F. J. (2000). Effects of subdural haematoma on sensorimotor functioning and spatial learning in rats. Neuropharmacology, 39, 817-834

Fisher, A., \& Hanin, I. (1986). Potential animal models for senile dementia of Alzheimer's type with emphasis on AF64A-induced cholinotoxicity. Annual Reviews of Pharmacology \& Toxicology, 26, 161-181.

Grigoryan, G., Hodges H., Mitchell S., Sinden J. D., \& Gray, J. A. (1996). 6-OHDA lesions of the nucleus accumbens accentuate memory deficits in animals with lesions to the forebrain cholinergic projection system: Effects of nicotine administration on learning and memory in the water maze. Neurobiology of Learning \& Memory, 65, 135-153.

Lermontova, N. N., Lukoyanov, N. V., Serkova, T. P., Lukoyanova, E. A., \& BAchurin, S. O. (1998). Effects of Tacrine on memory deficits in rats treated with cholinergic neurotoxin AF64A. Molecular \& Chemical Neuropathology, 33, 51-61.

MorRIS, R. G. M. (1981). Spatial localization does not require the presence of local cues. Learning \& Motivation, 12, 239-260.

SteEle, R. J., \& Morris, R. G. M. (1999). Delay-dependent impairment of a matching-to-place task with chronic and intrahippocampal infusion of the NMDA-antagonist D-AP5. Hippocampus, 9, 118-136.

WAlsh, T., \& Opello, K. (1994). The use of AF64A to model Alzheimer's disease. In M. I. Woodruff \& A. J. Nonneman (Eds.), Toxininduced models of neurological disorders (pp. 259-279). New York: Plenum.

Whishaw I. Q., CASSEl J.-C., \& JARRARdL. E. (1995). Rats with fimbriafornix lesions display a place response in a swimming pool: A dissociation between getting there and knowing where. Journal of Neuroscience, 15, 5779-5788.

(Manuscript received September 27, 2000; revision accepted for publication June 7, 2001.) 